\title{
Photoluminescence quenching in films of conjugated polymers by electrochemical doping
}

S. van Reenen, M.V. Vitorino, S.C. J. Meskers, R.A. J. Janssen and Martijn Kemerink

\section{Linköping University Post Print}

\section{Tweet}

N.B.: When citing this work, cite the original article.

Original Publication:

S. van Reenen, M.V. Vitorino, S.C. J. Meskers, R.A. J. Janssen and Martijn Kemerink, Photoluminescence quenching in films of conjugated polymers by electrochemical doping, 2014, Physical Review B. Condensed Matter and Materials Physics, (89), 20, 205206.

http://dx.doi.org/10.1103/PhysRevB.89.205206

Copyright: American Physical Society http://www.aps.org/

Postprint available at: Linköping University Electronic Press http://urn.kb.se/resolve?urn=urn:nbn:se:liu:diva-108168 


\title{
Photoluminescence quenching in films of conjugated polymers by electrochemical doping
}

\author{
S. van Reenen, ${ }^{1}$ M. V. Vitorino, ${ }^{1,2}$ S. C. J. Meskers, ${ }^{3}$ R. A. J. Janssen,,${ }^{1,3}$ and M. Kemerink ${ }^{1,4, *}$ \\ ${ }^{1}$ Department of Applied Physics, Eindhoven University of Technology, P.O. Box 513, 5600 MB Eindhoven, The Netherlands \\ ${ }^{2}$ Departamento de Fisica, Universidade de Lisboa, Campo Grando 1749-016, Lisboa, Portugal \\ ${ }^{3}$ Department of Chemical Engineering, Eindhoven University of Technology, P.O. Box 513, 5600 MB Eindhoven, The Netherlands \\ ${ }^{4}$ Department of Physics, Chemistry and Biology (IFM), Linköping University, SE-58183 Sweden \\ (Received 19 December 2013; revised manuscript received 13 May 2014; published 27 May 2014)
}

\begin{abstract}
An important loss mechanism in organic electroluminescent devices is exciton quenching by polarons. Gradual electrochemical doping of various conjugated polymer films enabled the determination of the doping density dependence of photoluminescence quenching. Electrochemical doping was achieved by contacting the film with a solid electrochemical gate and an injecting contact. A sharp reduction in photoluminescence was observed for doping densities between $10^{18}$ and $10^{19} \mathrm{~cm}^{-3}$. The doping density dependence is quantitatively modeled by exciton diffusion in a homogeneous density of polarons followed by either Förster resonance energy transfer or charge transfer. Both mechanisms need to be considered to describe polaron-induced exciton quenching. Thus, to reduce exciton-polaron quenching in organic optoelectronic devices, both mechanisms must be prevented by reducing the exciton diffusion, the spectral overlap, the doping density, or a combination thereof.
\end{abstract}

DOI: 10.1103/PhysRevB.89.205206

PACS number(s): 73.50.Gr, 73.61.Ph, 78.55.Kz

\section{INTRODUCTION}

Optimization of the external quantum efficiency (EQE) for charge to emitted photon conversion is presently one of the major challenges in improving organic light-emitting devices. At high luminance levels, the efficiency typically rolls off due to exciton quenching [1]. Excitons are electron-hole pairs with a luminescent lifetime dependent on the type of semiconductor and spin state that can be either a singlet or triplet. They are either formed by the absorption of a photon or by the meeting of injected electrons and holes. Excitons can be quenched in the presence of sufficiently large electric fields [2], by other excitons [3], or by polarons [4]. Which of these is dominant depends on the device and the emitter used.

Exciton quenching by polarons typically occurs in devices in which high carrier densities are present close to the region where excitons are formed. Devices with such high carrier densities are light-emitting electrochemical cells (LECs) [5], light-emitting field effect transistors (LEFETs) [6], or organic electrochemical light-emitting transistors (OECTs) [7]. In organic light-emitting diodes (OLEDs), exciton-polaron quenching becomes significant at high brightness, necessary for lighting and display applications [1]. Also in organic photovoltaic cells (OPV), exciton quenching is found to be an important loss process [4]. To address this loss process efficiently, it is necessary that the underlying mechanism is known. In literature, attention is mainly given to resonance energy transfer as the main loss mechanism [4,8-10]. This, however, does not exclude other competing loss processes like charge transfer (CT) [10,11].

Here, we present an experimental study of quenching of optically induced excitons by polarons introduced by controlled electrochemical doping $[12,13]$. The doping density was determined by the integration of the current and confirmed by bleaching of the light-emitting polymer. By this method, the doping dependence of exciton quenching was determined

\footnotetext{
*martijn.kemerink@liu.se
}

for several prototypical conjugated polymers. The doping dependence was then analytically described by two alternative processes: (1) exciton diffusion followed by Förster resonance energy transfer (FRET) to the polaron associated with the dopant or (2) exciton diffusion followed by CT between the polaron and the exciton. As both models successfully fit the data, both processes can account for the observed exciton-polaron quenching. Doping densities at which quenching occurs range between $10^{18}$ and $10^{19} \mathrm{~cm}^{-3}$, setting an upper limit for the doping, or charge density at or near the recombination zone in organic optoelectronic devices.

\section{EXPERIMENT}

The devices in this study consist of a bilayer of a conjugated polymer and an electrolyte sandwiched between an indium tin oxide (ITO) and a Au electrode. A schematic of the device is shown in Fig. 1(a). The conjugated polymers are either (i) phenyl-substituted poly(p-phenylene vinylene) copolymer (SY-PPV, Merck, catalog number PDY-132), commonly termed "Super Yellow", (ii) poly[2-methoxy5-(3',7'-dimethyloctyloxy)-1,4-phenylenevinylene] (MDMOPPV, $M_{w}>1 \times 10^{6} \mathrm{~g} \mathrm{~mol}^{-1}$, American Dye Source), or (iii) poly(3-hexylthiophene) (P3HT, $>98 \%$ head to tail, $M_{n}=54000-75000 \mathrm{~g} \mathrm{~mol}^{-1}$, Plextronics, purchased from Aldrich). The PPV variants and P3HT were deposited by first dissolving them in chloroform or chlorobenzene, respectively, to obtain $10 \mathrm{mg} \mathrm{ml}^{-1}$ solutions, followed by spin coating to yield $\sim 100 \mathrm{~nm}$ films on top of cleaned ITO substrates. The electrolyte is a mixture of poly(ethylene oxide) (PEO, $M_{w}=5 \times 10^{5} \mathrm{~g} \mathrm{~mol}^{-1}$, Aldrich) and potassium triflate $\left(\mathrm{KCF}_{3} \mathrm{SO}_{3}, 98 \%\right.$, Aldrich) at a weight ratio of $5: 1$. The electrolyte was drop coated from acetone on top of the polymer film, which was placed on a hot plate at $T=45^{\circ} \mathrm{C}$. The thickness of the solid electrolyte droplet was $2-3 \mu \mathrm{m}$. Gold electrodes were subsequently thermally deposited in a deposition chamber in a glove box under a vacuum of $\sim 10^{-6}$ mbar. The thickness of the gold layer was $100 \mathrm{~nm}$. Alternatively, to enable absorption measurements by transmission, a $30 \mathrm{~nm}$ 
(a)

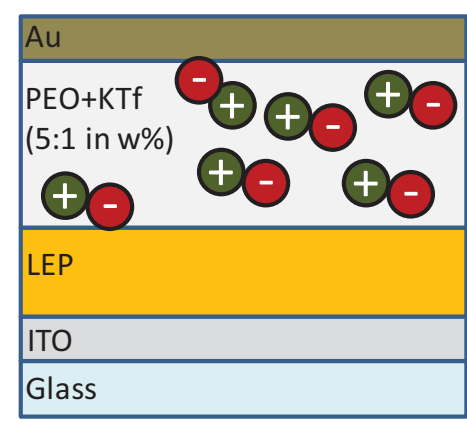

(b)

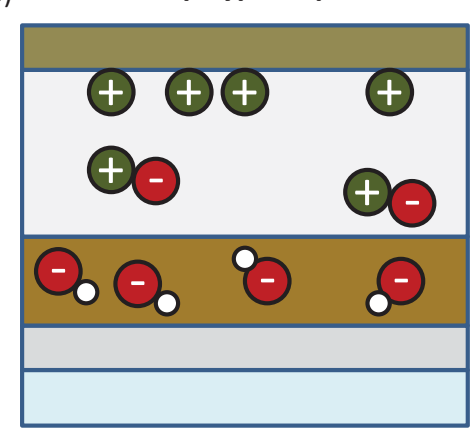

(c)

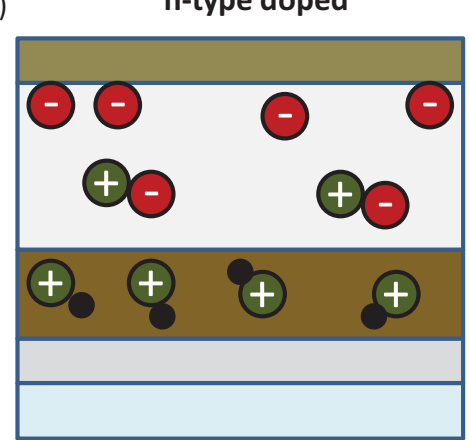

FIG. 1. (Color online) (a) Schematic device layout for electrochemical doping of light-emitting polymers (LEP). The same schematic after p- and n-type electrochemical doping is shown in (b) and (c), respectively. Red, green, white, and black dots represent anions, cations, holes, and electrons, respectively.

semitransparent gold electrode was used. The active area of the device, defined by a shadow mask, was $0.161 \mathrm{~cm}^{2}$. All device manufacturing was done under a nitrogen atmosphere.

Current-voltage characteristics were measured by a Keithley 2400 SourceMeter. For the detection of photoluminescence (PL) during electrochemical doping, an Edinburgh timeresolved fluorescence spectrometer FLS920 was used. For these measurements, the device was put in a closed nitrogenfilled box with a glass window. For the detection of the absorption during electrochemical doping, a Shimadzu UV1601PC UV/visible scanning spectrophotometer was used. For these measurements the device was put in a closed nitrogenfilled box with two windows to allow for measurements in transmission. Absorption measurements were also performed by measurement of the reflection. In this case, a device with a $100 \mathrm{~nm}$ gold top electrode was used.

\section{EXPERIMENTAL RESULTS}

To investigate the polaron density dependence of luminescence quenching in conjugated polymers, a solid-state electrochemical cell was fabricated as described above. A schematic of the device layout is shown in Fig. 1(a). The device contains a $100 \mathrm{~nm}$ film of conjugated polymer. On the bottom side, this film is contacted by a transparent ITO electrode to allow for electronic charge carrier injection. The top side of the film is electrochemically gated to allow ionic charge carrier injection. Dependent on the sign of the applied bias voltage, the cell was electrochemically n- or p-type doped. Schematic illustrations of both types of doping are drawn in Figs. 1(b) and 1(c). This doping can be viewed as electrostatic stabilization of injected electronic charges in the conjugated polymer by injected anions or cations. Note, in this context, the strong similarity to the dynamic doping process occurring in LECs $[14,15]$.

To determine the doping density dependence of the exciton quenching in the conjugated polymer films, two experiments were performed simultaneously: (i) the change in the PL of the illuminated film was measured, and (ii) the polymer film was gradually, electrochemically doped. To confirm that doping of the conjugated polymer indeed took place, the optical absorption was monitored in an additional experiment.
Prior to the experiment, PL excitation and emission spectra were determined for the different polymer films (Fig. 2). The wavelengths at which the spectra show a peak were used as the excitation and emission wavelength during the transient quenching experiment. To compensate for the background emission, an ITO-electrolyte-Au device was prepared and characterized at the same emission and excitation wavelengths. During the doping experiments that are explained below, the normalized change in PL was determined as well. In Fig. 3, it is indicated by the solid line. As the doping progresses in time, the PL is found to decrease.

Regarding the doping experiments, a stepwise increasing bias voltage was applied to the contacts as shown by the dashed line in Fig. 3. Each bias level was maintained for approximately $300 \mathrm{~s}$ to allow doping to saturate. A typical trace of the current passing through the cell is indicated on a logarithmic scale by the dotted line in Fig. 3. After each bias voltage step, the current is observed to quickly increase, followed by a slow decrease towards a constant value. This constant value is attributed to leakage and cannot be related to ongoing electrochemical doping: ions are blocked by the ITO electrode, and electronic carriers are blocked by the electrolyte, so the electronic current should ultimately vanish when maintaining a constant bias

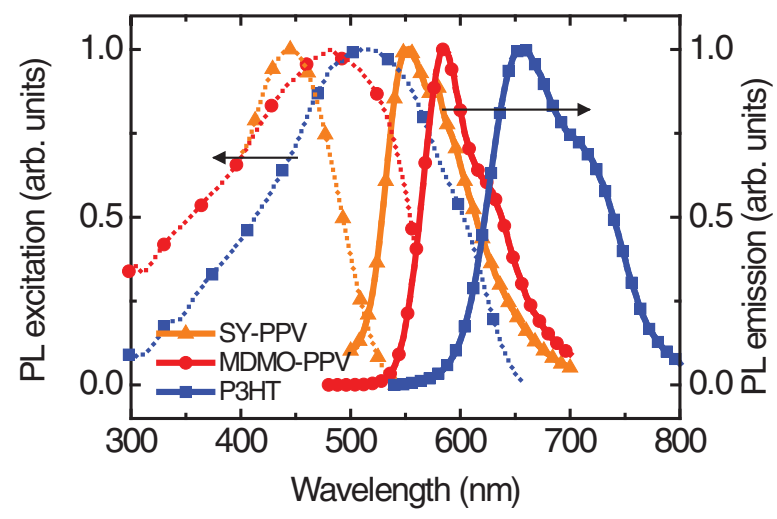

FIG. 2. (Color online) Photoluminescence-excitation and PLemission spectra of Super Yellow PPV, MDMO-PPV, and P3HT. For the PL excitation measurements, a detection wavelength of $550 \mathrm{~nm}$ was used. For the PL emission measurements, an excitation wavelength of $440 \mathrm{~nm}$ was used. 


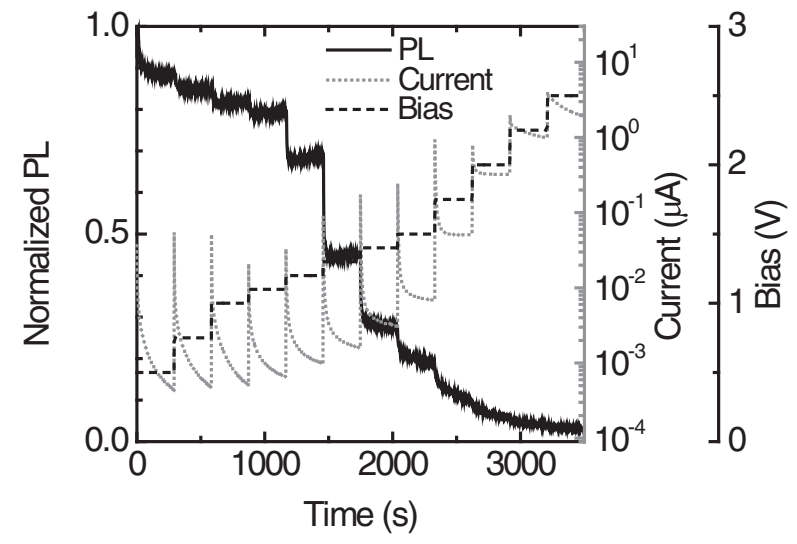

FIG. 3. Photoluminescence (solid line), applied bias voltage (dashed line), and current (dotted line) transients during oxidation of SY-PPV. The device was excited at $\lambda=440 \mathrm{~nm}$. Photoluminescence was detected at $\lambda=550 \mathrm{~nm}$.

voltage. This interpretation is substantiated by the stabilization of the PL quenching signal (solid line) after each voltage step on the same timescale as on which the current stabilizes. The fact that the PL signal does not completely stabilize indicates either a slow redistribution of doping or ongoing, slow injection of ions into the semiconductor. Leakage currents may be related to Au diffused into the electrolyte, forming conducting pathways. However, for example, in Ref. [16], we have observed a penetration of (more reactive) Cs into a soft polymer layer by only $\sim 15 \mathrm{~nm}$. The much lower reactivity of Au may, however, increase the penetration depth. By subtracting the leakage current from the overall current at each bias voltage, the doping current is determined. Integration of this current with respect to time then gives the amount of doping put into the $100 \mathrm{~nm}$ film. By dividing by the volume of the film, the doping density is obtained. As the leakage current can only be estimated, an error in the doping density arises which may approximately be a factor 2 , as deduced from repetitive measurements (see Fig. S1 in the Supplemental Material [17]).

During doping of the conjugated polymer, the same amount of oppositely charged ions is transported towards the electrolyte-electrode interface and must be electrostatically compensated. We assume an electric double layer (EDL) formation of $1 \mathrm{~nm}$ at a completely flat interface would give rise to a voltage drop of $\sim 1 \mathrm{~V}$ when we account for the electrode area being $\sim 3$ times larger than the semiconductor. This value is not unreasonable given that the maximum applied bias voltage of $3 \mathrm{~V}$ is larger than expected to be needed to fully dope the organic semiconductor. Other factors contributing to the absence of large voltage drops at the electrolyteelectrode interface may be related to a large irregular surface area induced by diffusion of $\mathrm{Au}$ into the electrolyte during deposition [16] and diffusion of ions through the Au electrode during the experiment, as reported before by Matyba et al. [14].

Combination of the results in Fig. 3 allowed characterization of the doping density dependence of the PL quenching process. This was done similarly for $\mathrm{n}$ - and $\mathrm{p}$-type doping of SY-PPV as well as for p-type doping of MDMO-PPV and P3HT. The results are shown in Fig. 4. The vertical

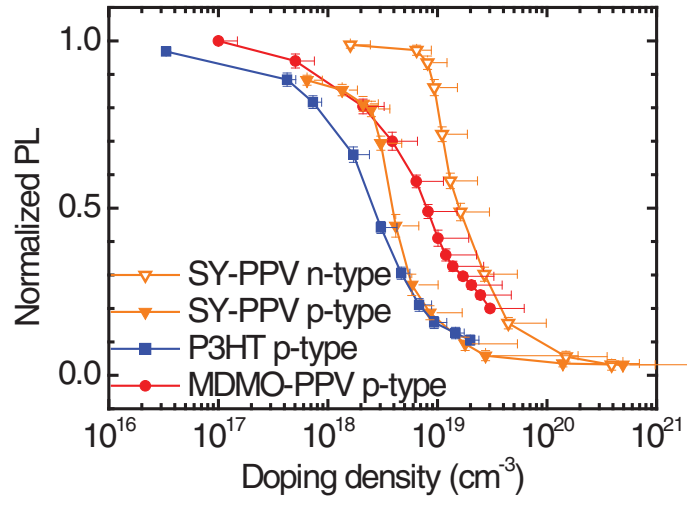

FIG. 4. (Color online) Photoluminescence quenching as a function of doping density for SY-PPV, MDMO PPV, and P3HT.

error bars are related to the PL signal not being stable yet. In each case a relatively strong decrease in PL emission occurs at doping densities between $10^{18}$ and $10^{19} \mathrm{~cm}^{-3}$, which corresponds to doping levels of approximately $0.1 \%$ and $1.0 \%$ when assuming a density of states of $10^{21} \mathrm{~cm}^{-3}$ [18]. The doping density is determined by integrating the current after correction for the leakage current. The magnitude of the leakage current is, however, uncertain. Therefore, the doping density was also calculated without accounting for the leakage current. The relative contribution of leakage and doping current (as shown in Fig. S1(b) in the Supplemental Material [17]) shows that the doping and leakage current increase roughly in parallel. This limits the relative error in the extracted doping concentration, which is the important quantity in the present discussion. To quantify the effects of the leakage current, we added corresponding error margins in Fig. 4. The indicated upper limit is given by the absolute maximum of possible doping density in case we correct for no or an underestimated (for P3HT) leakage current. The data point itself is based on the (over)estimated leakage current and forms, therefore, a lower limit. A reference measurement on a cell without organic semiconductor is included in Fig. S2 in the Supplemental Material [17]. Neither indications of side reactions were observed in the measured current nor any change in color. More importantly, the currents found here are approximately an order of magnitude below the currents observed in the full device, i.e. with the semiconductor present, indicating that the latter is dominated by doping and not by leakage, in line with our arguments above. In more detail (see Fig. S2 in the Supplemental Material [17]), we calculated the associated charge densities by integration of the leakage currents. The integrated carrier density in the devices with organic semiconductor is at least larger by an order of magnitude than without the semiconductor, demonstrating that the extracted doping densities are maximally overestimated by $10 \%$.

For accurate determination of the doping density, a homogeneous doping distribution is required. The incomplete stabilization of the PL signal observed in Fig. 3 suggests that doping redistributes. Likely, ions are injected in the film in a filamentary or otherwise inhomogeneous manner, causing strong quenching in those regions. However, the corresponding density gradients are unstable, and a redistribution of ions will 
(a)

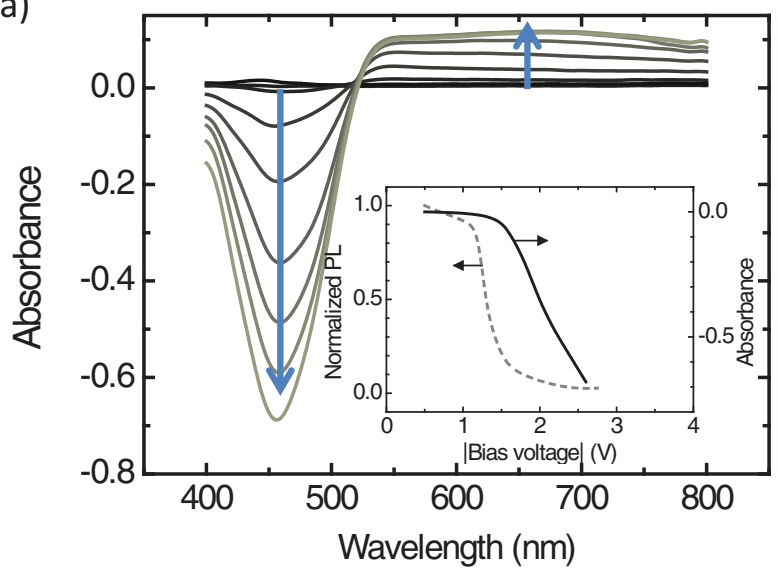

(b)

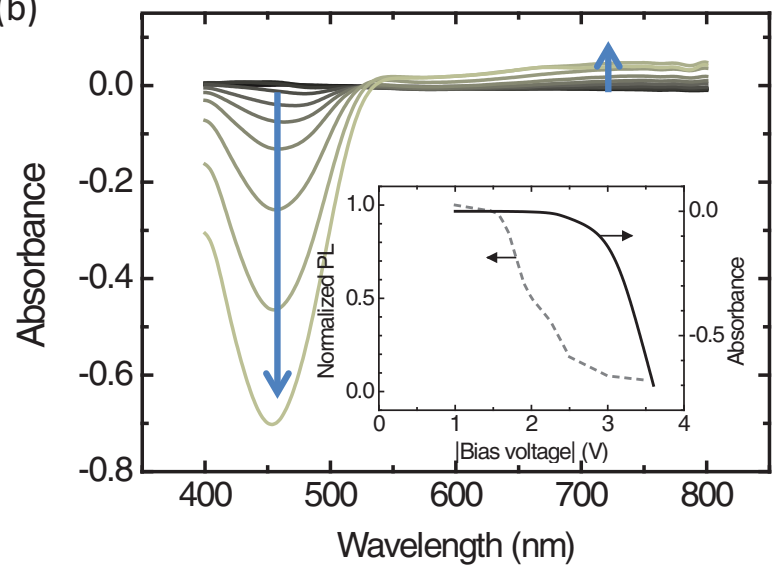

FIG. 5. (Color online) Absorbance of (a) p- and (b) n-type doping in SY-PPV at different doping levels. The absorbance by doping is determined by subtracting the absorbance at any bias voltage by the absorbance of the unbiased cell: $A_{\text {doping }}=A\left(V_{\text {bias }}\right)-A\left(V_{\text {bias }}=0\right)$. A semitransparent Au top electrode was used with a thickness of $30 \mathrm{~nm}$. The blue arrows indicate the change in absorbance for larger doping densities, starting at zero doping. The insets show the normalized PL (gray dashed) and the absorbance (black solid) by doping as a function of applied bias.

follow, lowering the maximum concentrations and reducing the quenching, provided the resulting overall doping level is sufficiently low. The tendency of ions to redistribute is related to drift and diffusion, both in favor of a homogeneous distribution. Another indirect indication that the doping is relatively homogeneous is the abrupt reduction of PL versus doping density as shown in Fig. 4 for SY-PPV. Inhomogeneity, i.e. the formation of doped and undoped domains, would correspond to a more gradual reduction of the PL signal. The absence of substantial inhomogeneity is finally substantiated by optical inspection of the device darkening during doping, which was found to be homogeneous within the experimental resolution of $\sim 100 \mu \mathrm{m}$.

Comparison between $\mathrm{p}$ - and n-type doping in SY-PPV demonstrates that p-type doping quenches at lower carrier densities. This is in line with observations in planar LECs. In such cells, both types of doping are present simultaneously. The n-type doped regions of PPV are typically observed to quench less than the p-type doping regions [15]. Here, we show that this behavior can at least partially be explained by stronger PL quenching by p-type doping. A comparison between P3HT and MDMO-PPV shows only small differences in shape and critical doping density of PL quenching by p-type doping. $\mathrm{N}$ type doping in MDMO-PPV and P3HT occurred at relatively large bias voltages at which electrochemical reactions with the electrolyte distort the measurements. Therefore, these measurements were not included. The functional shape of the quenching curve of P3HT and MDMO-PPV seems to be different from that of SY-PPV. We return to this at the end of the Discussion section.

Next to the effect of PL quenching induced by doping, the absorption spectrum of the dopants was also measured in SY-PPV. The change in absorbance by doping of SYPPV is plotted in Figs. 5(a) and 5(b) for p- and n-type doping, respectively. The negative differential absorbance at $\lambda=460 \mathrm{~nm}$ is at the same position as the absorption spectrum of SY-PPV (dotted orange line in Fig. 2). The effect is, therefore, related to bleaching: doping fills the density of states, reducing the number of sites available for the formation of excitons [12]. This bleaching is an important observation as it indicates filling of the density of states of the light-emitting polymer. The enhanced transmission due to ground state bleaching of $\Delta T / T \approx 2.1$ [see Fig. $5(\mathrm{a})$; the trace where a bleaching peak at $\lambda=460 \mathrm{~nm}$ is observed of $A=-0.5$, where $\left.\Delta T / T=10^{-A}-1\right]$ was measured at an electrically determined charge density between $5 \cdot 10^{20}$ and $3 \cdot 10^{21} \mathrm{~cm}^{-3}$. To estimate whether these values are reasonable, we made a comparison with values obtained in Refs. [19,20], where a relative enhancement in transmission by $6 \cdot 10^{-4}$ was observed at a (photoexcited) charge density of $1 \cdot 10^{18} \mathrm{~cm}^{-3}$. Making the reasonable assumption that bleaching is linear in density, densities of $5 \cdot 10^{20}$ and $3 \cdot 10^{21} \mathrm{~cm}^{-3}$ would then lead to $\Delta T / T$ between 0.3 and 1.8 , respectively. This value is in more than reasonable agreement with the $\Delta T / T \approx 2.1$ we measure, especially since we ignore differences, for example, in site density between the materials. This confirms that electrochemical doping of the conjugated polymer indeed takes place and that the calculated doping density by the integration of the current is reliable.

To determine whether the PL quenching in Fig. 4 originates from the reduced optical density as observed in Fig. 5, the relative absorbance and the normalized PL are plotted in the same graph in the insets in Figs. 5(a) and 5(b). For both pand n-type doping of SY-PPV, bleaching sets in clearly after the majority of PL has already been quenched. Hence, PL quenching and absorption bleaching are independent processes in this respect. At larger wavelengths, in Figs. 5(a) and 5(b), absorption bands appear for both $n$ - and p-type doping. These bands sit partially at the same wavelength as the PL spectrum of SY-PPV (solid orange line in Fig. 2) and are attributed to polaron absorption [12].

\section{DISCUSSION}

To interpret the results shown in Fig. 4, first the effect of applying a bias voltage to the device [see Fig. 1(a)] needs to be 


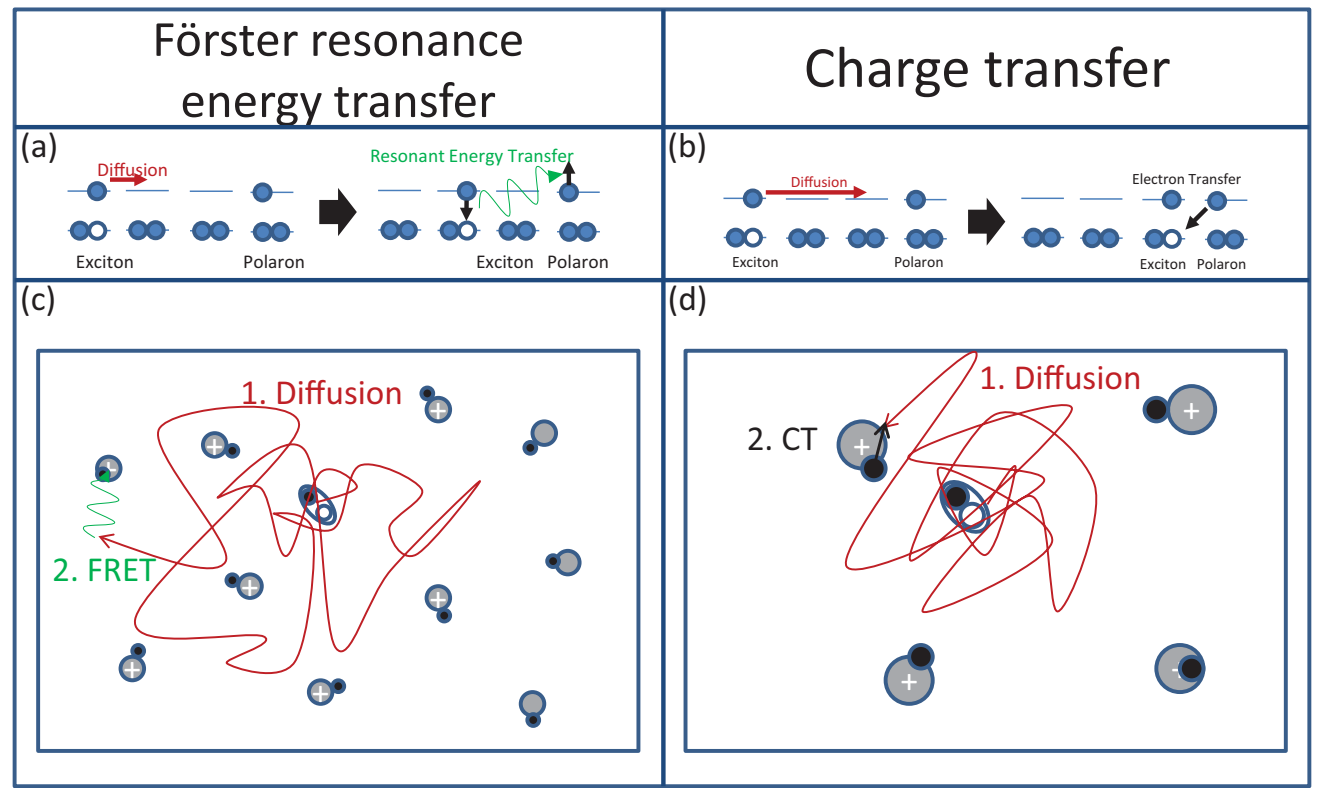

FIG. 6. (Color online) Schematic representation of the considered exciton quenching mechanisms in a random distribution of doping sites: (a) and (c) FRETr and (b) and (d) CT. Both mechanisms are preceded by exciton diffusion.

understood. Initially, the PEO is filled with ions, whereas the PPV is empty. The difference in chemical potential of the ions in both layers is expected to be strongly in favor of the PEO film due to its chemical structure. This difference forms an ion injection barrier which impedes ion injection into the PPV film. This difference across the PEO-PPV interface is, however, opposed by (i) a difference in ion densities resulting in a diffusion current and (ii) a difference in electrostatic potential due to the bias voltage applied to the contacts. Increasing the applied bias voltage results in an increased ion density at the interface, enlarging both the potential drop and the density gradient at the interface. This, in turn, drives ions into the semiconductor. In the experiment, the bias voltage is increased stepwise and ion injection is observed at sufficiently large voltages. $\mathrm{K}^{+}$or $\mathrm{Tf}^{-}$injection in SY-PPV are observed to start at $\left|V_{\text {bias }}\right| \approx 1.6 \mathrm{~V}$ and $1.2 \mathrm{~V}$ for $\mathrm{n}$ - and p-type doping, respectively, see insets in Figs. 5(a) and 5(b).

The exciton quenching process in Fig. 4 is the result of an interaction between a polaron, stabilized by an ionic dopant, and an exciton. No significant external electric field, which could also contribute to exciton quenching, is expected within the SY-PPV layer: the boundary conditions induced by the electrode and the electrolyte result in a homogeneous pile-up of ionic and electronic carriers in the SY-PPV film. Once injected by, respectively, the electrolyte or the electrode, the carrier cannot leave the film through the other contact. This means that, ultimately, the net electronic and ionic current must be zero. The net current is determined by drift and diffusion, which should thus cancel or both be equal to zero. As the doping mechanism leads to electronic and ionic carriers having the same density profile, diffusion is in the same direction for both types of carriers. Drift, however, is in opposite directions for both types of carriers due to the oppositeness of the particles' charge. Hence, to obtain a zero net electronic and ionic current, the drift and diffusion components of the ionic dopants and the polarons must both be zero. The zero diffusion current implies that constant concentrations for electronic and ionic carriers are present. These qualitative arguments are confirmed by numerical drift-diffusion simulations shown in Fig. S3 in the Supplemental Material [17].

Regarding exciton quenching by polarons, two alternative processes shall be considered: FRET and CT. Other alternatives like multipolar interactions [21] were not considered. Diffusion of the exciton through the film prior to quenching must, however, be accounted for. Polaron diffusion was found to be negligible compared to exciton diffusion, as will be justified below in Sec. IV C. In Fig. 6(a), schematic representation is given of both models. In the next two paragraphs, these models are discussed in detail and will be related to our experimental findings.

\section{A. Förster resonance energy transfer}

Resonance energy transfer is an energy transfer between a donor and an acceptor [22]. In our case, the acceptor is an electron- or hole-polaron that is excited to a higher state, as illustrated in Fig. 6(a). Since the donor loses its excitation by to the acceptor by the coupling of transition dipoles, it is a nonradiative loss process. Spectral overlap, however, is required between the emission spectrum of the donor and the excitation spectrum of the acceptor to make the process efficient. The spectra in, respectively, Figs. 2 and 5 show that this is indeed the case in SY-PPV for exciton emission and polaron excitation. In the next subsections, two possible scenarios for FRET are explained.

\section{Single pair of fixed dipoles}

The most basic description of FRET is between a donor and an acceptor that are two dipoles, fixed in their relative orientation and position. The transfer rate constant derived by 
Förster is expressed as

$$
k_{T}=\frac{1}{\tau_{D}^{0}}\left[\frac{R_{0}}{r}\right]^{6}
$$

where $\tau_{D}^{0}$ is the lifetime of the donor in absence of energy transfer, $r$ is the distance between donor and acceptor, and $R_{0}$ is the critical distance at which the probability of transfer and decay of the donor is equal. The Förster radius $R_{0}$ can be determined experimentally and is expressed as

$$
R_{0}^{6}=\frac{9000(\ln 10) \kappa^{2} \Phi_{D}^{0}}{128 \pi^{5} N_{A} n^{4}} \int_{0}^{\infty} I_{D}(\lambda) \varepsilon_{A}(\lambda) \lambda^{4} d \lambda,
$$

where $\kappa^{2}$ is the orientational factor, $\Phi_{D}^{0}$ is the fluorescence quantum yield of the donor without transfer, $n$ is the average refractive index of the medium, $I_{D}(\lambda)$ is the normalized fluorescence spectrum of the donor, and $\varepsilon_{A}(\lambda)$ is the molar absorption coefficient of the acceptor.

In our case, this description of FRET does not apply as the formed exciton is likely to be mobile and is excited in a film filled with multiple acceptors. Therefore, exciton diffusion and multiple acceptors need to be taken into account for a correct description.

\section{Exciton diffusion with multiple acceptors}

The exciton quenching process can be described by diffusion with a sink term $k(r)=a / r^{6}$ with the correct distance dependence between the donor and acceptor $r$ [22,23]. The donor and acceptor are approximated as point dipoles, i.e. excluding delocalization of the polaron and exciton. For excitons, a delocalization over several monomer subunits may be expected (e.g. $\sim 1 \mathrm{~nm}^{24}$ ). Polarons compensated by ions at relatively low doping levels of $\sim 1 \%$ are more localized due to the ionic Coulomb trap (i.e. $<1 \mathrm{~nm}$ ) [13]. The diffusion equation then becomes

$$
\frac{\partial C_{E}(r, t)}{\partial t}=D \nabla^{2} C_{E}(r, t)-k(r) C_{E}(r, t),
$$

where $C_{E}$ is the exciton density and $D$ the exciton diffusion coefficient. Gösele et al. [23] found the following approximate solution to this problem:

$$
k_{T}(t)=4 \pi R_{\mathrm{eff}} D\left[1+\frac{R_{\mathrm{eff}}}{(\pi D t)^{1 / 2}}\right],
$$

where

$$
R_{\text {eff }}=0.676\left(\frac{R_{0}^{6}}{\tau_{D}^{0} D}\right)^{1 / 4} .
$$

The fluorescence decay after excitation can then be described by

$$
\begin{aligned}
\frac{i(t)}{i(0)} & =\exp \left\{\frac{1}{\tau_{D}^{0}}+C_{A} \int_{0}^{t} k_{T}\left(t^{\prime}\right) d t^{\prime}\right\} \\
& =\exp \left(-\frac{1}{\tau_{D}^{0}} t-4 \pi R_{\mathrm{eff}} D C_{A} t-2 \cdot 4 \sqrt{\pi D} R_{\mathrm{eff}}^{2} C_{A} \sqrt{t}\right),
\end{aligned}
$$

where $C_{\mathrm{A}}$ is the density of the absorbing species. The ratio in fluorescence quantum yield with $\left(\Phi_{D}\right)$ and without $\left(\Phi_{D}^{0}\right)$ the presence of a quencher then becomes:

$$
\frac{\Phi_{D}}{\Phi_{D}^{0}}=\frac{\int_{0}^{\infty}\left[\exp \left(-\frac{1}{\tau_{D}^{0}} t-4 \pi R_{\mathrm{eff}} D C_{A} t-2 \cdot 4 \sqrt{\pi D} R_{\mathrm{eff}}^{2} C_{A} \sqrt{t}\right)\right] d t}{\int_{0}^{\infty}\left[\exp \left(-\frac{1}{\tau_{D}^{0}} t\right)\right] d t}
$$

To fit Eq. (7) to the experimental data, the following values were taken from literature: $k_{\text {decay }}=1 / \tau_{D}^{0}=1.54 \mathrm{~ns}^{-1}$ found in SY-PPV and $D=3 \cdot 10^{-4} \mathrm{~cm}^{2} \mathrm{~s}^{-1}$ found in the related NRS-PPV [26,27]. These fits are shown in Fig. 7(a) (blue solid lines) for different values of $R_{0}$ ranging from 1 to $4 \mathrm{~nm}$. The dashed red lines indicate similar fits with the only difference that $D=0 \mathrm{~cm}^{2} \mathrm{~s}^{-1}$ to illustrate the effect of resonant energy transfer quenching of immobile excitons excited in a semiconductor that is homogeneously filled with polarons. As expected, diffusion significantly enhances the likelihood of quenching. As quenching occurs at distances $(\sim 2.5 \mathrm{~nm})$ larger than the size of an exciton delocalized over several monomer subunits $(\sim 1 \mathrm{~nm})$, the point dipole approximation gives a reasonable estimation of the polaron-induced exciton quenching with an error margin of $1 \mathrm{~nm}$.

\section{B. Charge transfer}

To derive an expression of the fluorescence quantum yield of the donor as a function of the doping density, the following simple model was used. An exciton diffuses stepwise through the semiconductor. After each step, the exciton has a probability to decay radiatively $P_{\text {decay }}$ or a probability $P_{\text {quench }}$ to arrive next to a polaron, which results in quenching of the exciton when the polaron recombines with the charge of opposite polarity in the exciton, as illustrated in Figs. 6(b) and $6(\mathrm{~d})$. Here, we assume that polarons and excitons can only occupy one site and that quenching always takes place when the exciton is next to a polaron as CT is a relatively fast process [24]. By comparison of these probabilities, the probability of exciton quenching can be determined as a function of doping density.

The exciton quenching probability after one diffusion step can be related to the ratio of doped site density $n_{\text {doping }}$ and the density of states $n_{\text {DOS }}$ :

$$
P_{\text {quench }}=A \frac{n_{\text {doping }}}{n_{\text {DOS }}},
$$

where $A$ is the average number of new neighboring sites that can quench the exciton. The value of $A$ depends on the coordination of the excitons and polarons. In the first instance, a one-dimensional system is assumed for the polymer system. To determine the quenching probability as a function of doping density, $n_{\text {DOS }}=6 \cdot 10^{26} \mathrm{~m}^{-3}$ and $A=2$ were taken [18]. 
(a)

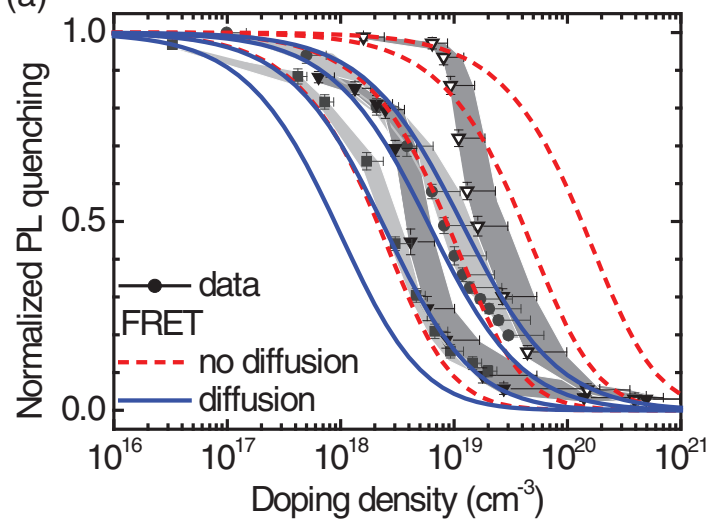

(b)

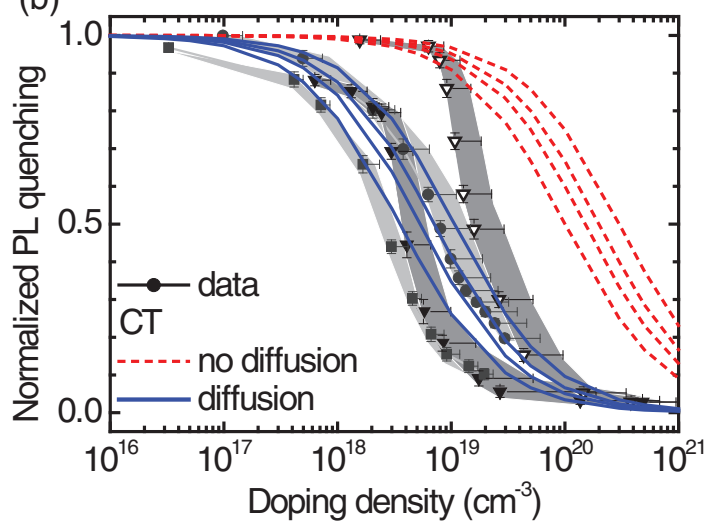

FIG. 7. (Color online) Fits (lines) of the PL quenching data (symbols + shaded regions representing the error in the data) of n-type doping of SY-PPV (down triangles) and p-type doping of SY-PPV (up triangles), MDMO-PPV (squares) and P3HT (dots). (a) Fits following the resonance energy transfer model without diffusion are dashed red (see Eq. (7); $D=0 \mathrm{~cm}^{2} \mathrm{~s}^{-1} ; R_{0}=4,2.5,1.5,1.0 \mathrm{~nm}$ from left to right) and with diffusion solid blue (see Eq. (7); $D=3 \cdot 10^{-4} \mathrm{~cm}^{2} \mathrm{~s}^{-1} ; R_{0}=4,2.5,1.5,1.0 \mathrm{~nm}$ from left to right). (b) Fits following the CT model without diffusion dashed red (see Eq. (10); $D=0 \mathrm{~cm}^{2} \mathrm{~s}^{-1} ; A=2,3,4,6$ neighbors from left to right) and with diffusion solid blue (see Eq. (10); $D=3 \cdot 10^{-4} \mathrm{~cm}^{2} \mathrm{~s}^{-1} ; A=2,3,4,6$ neighbors from left to right).

Assuming physical contact between exciton and charge is needed for quenching, $n_{\mathrm{DOS}}{ }^{-1}$ equals the mean volume of a site-we assume excitons and polarons sit on sites of equal volume. Clearly, this is a gross simplification as some tunneling is likely to be relevant to quenching, implying the presence of a spatial gap and thus a reduced site volume. Here, $n_{\text {DOS }}{ }^{-1}$ is thus a coarse upper limit to the real exciton and polaron volumes. The used value for $n_{\text {DOS }}, 6 \cdot 10^{26} \mathrm{~m}^{-3}$, puts an upper limit just above $1 \mathrm{~nm}^{3}$, consistent with the length scales of a delocalized exciton [25].

The radiative decay rate of a single exciton was set to a fixed rate $k_{\text {rad,decay }}$. For an ensemble of excitons, this gives rise to an exponential radiative decay with a radiative decay rate $k_{\text {rad,decay }}$ when exciton-exciton interactions are ignored. The time of a single diffusion step is set equal to the time needed to overcome the distance of one neighboring site $r=n_{\mathrm{DOS}}^{-1 / 3}$ by diffusion. The diffusion length $r$ is, in turn, related to the exciton diffusion constant $D$ as $r=\sqrt{2 D t_{\text {step }}}$. The radiative decay probability during this single diffusion step can then be described by

$$
P_{\text {rad,decay }}=1-\exp \left(-\frac{k_{\text {rad,decay }}}{2 D \cdot n_{\mathrm{DOS}}^{2 / 3}}\right) .
$$

To determine the radiative decay probability, the following values were taken from literature: $k_{\text {rad,decay }}=1.54 \mathrm{~ns}^{-1}$ and $D=3 \cdot 10^{-4} \mathrm{~cm}^{2} \mathrm{~s}^{-1}[26,27]$.

Under the assumption that initial and final sites are independent the fluorescence quantum yield can then be determined by

$$
\frac{\Phi_{D}}{\Phi_{D}^{0}}=\frac{P_{\text {rad,decay }}}{P_{\text {quench }}+P_{\text {rad,decay }}} .
$$

A fit of this function for the given parameters is shown in Fig. 7(b) by the solid blue lines for $A=6,4,3,2$ neighbors. The dashed red lines indicate similar fits with the only difference that $D=0 \mathrm{~cm}^{2} \mathrm{~s}^{-1}$ to illustrate the effect of CT quenching of immobile excitons excited in a semiconductor that is homogeneously filled with polarons. An alternative approach to determine the effect of CT on the fluorescence quantum yield is by using Eq. (3) with a sink term $k \sim \exp (-r / b)$ [10,28], which must be solved numerically. An analytical expression can, however, be found when solving Eq. (3) for $k=0$ and the boundary condition that the probability of exciton quenching equals 1 when the exciton is inside a sphere with radius $R_{c}$ around the acceptor [22]. In Fig. S5 in the Supplemental Material [17], a fit based on this model is shown for $R_{c}=\frac{1}{2} n_{\mathrm{DOS}}^{-1 / 3}, k_{\mathrm{rad} \text {,decay }}=1.54 \mathrm{~ns}^{-1}$ and $D=3 \cdot 10^{-4} \mathrm{~cm}^{2} \mathrm{~s}^{-1}[26,27]$. The fit is qualitatively similar to the fits based on Eq. (10).

\section{Comparison of FRET and CT}

Comparison between the fits related to FRET and CT in Fig. 7 shows that there is little qualitative difference in the doping dependence between the two quenching mechanisms. This demonstrates that instead of the actual quenching model, FRET or CT, the homogeneous density of quenchers determines the qualitative doping density dependence of the exciton quenching. In fact it is not at all unlikely that both processes, CT and FRET, take place simultaneously. Given the fact that the doping density dependence is slightly different for both models, the relative contributions of both processes would also be dependent on the doping density. Regarding diffusion, also polaron diffusion may play a role in exciton quenching. This is, however, only the case for high mobility semiconductors like P3HT. The exciton diffusion itself can also be expected to be doping density dependent. An indication for this is discussed below.

The data that is qualitatively fit by both models is that of the p-type doping in MDMO-PPV and P3HT. The shift towards a lower doping density observed for P3HT can be related to the larger exciton diffusion coefficient in P3HT [29]: $1.8 \cdot 10^{-3}$ $\mathrm{cm}^{2} \mathrm{~s}^{-1}$ compared to $3.2 \cdot 10^{-4} \mathrm{~cm}^{2} \mathrm{~s}^{-1}$ in MDMO-PPV [30]. The exciton lifetime in both materials is similar $[31,32]$. It should be noted that, given the exciton lifetime and the exciton 
(a)

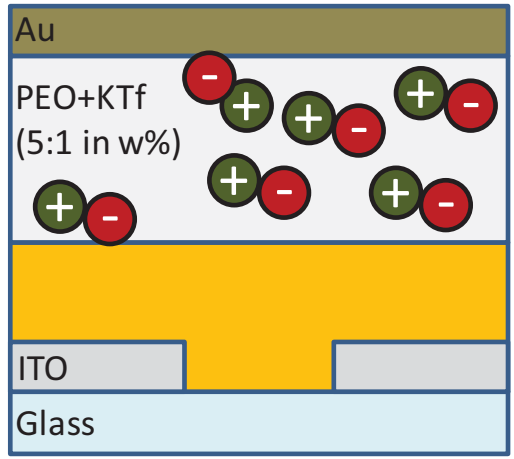

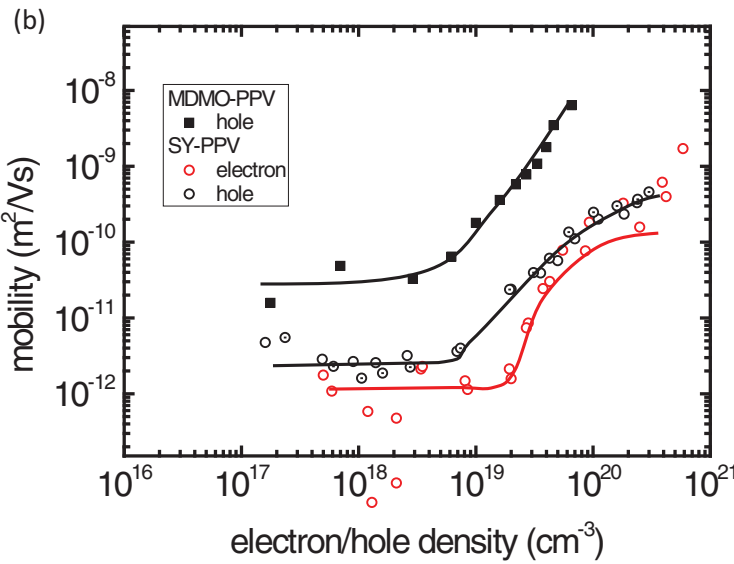

FIG. 8. (Color online) (a) Schematic device layout of the electrochemical transistor. (b) Electronic carrier mobility as a function of doping density. Lines were added that serve as guides to the eye to visualize the density threshold after which the mobility is observed to increase significantly.

diffusion coefficients of P3HT and MDMO-PPV, a relatively small critical radius of $\sim 1-1.5 \mathrm{~nm}$ is needed to quantitatively fit FRET in Fig. 7(a). The error in experimentally determined doping density of roughly a factor 2 results in an uncertainty in critical radius of $0.5 \mathrm{~nm}$. By measurement of the absorption spectrum in reflectance of p-type doping in MDMO-PPV (see Fig. S4(a) in the Supplemental Material [17]), $R_{0}$ could be calculated from Eq. (2). To this purpose the emission spectrum as shown in Fig. 2 was used. The orientation factor $\kappa^{2}$ was taken to be 0.655 [33], and the refractive index $n$ is estimated to be 2 [34]. Note that $\kappa^{2}$ can possibly range between 0 and 4 . In case the donor and emitter dipole lie in a plane, $\kappa^{2}$ equals 0.655 [22,33]. A fluorescence quantum yield of $8 \%$ was taken from Ref. [35]. The resultant Förster radius for p-type doping in MDMO-PPV was found to be approximately $3.0 \pm 0.3 \mathrm{~nm}$. This value is larger than the fitted value of $\sim 1.5 \mathrm{~nm}$ in Fig. 7(b). However, the factor $\sim 2$ difference is well within the margins induced by the uncertainties in the assumed exciton lifetime and diffusion coefficient, in the orientation factor and in the measured doping density.

The data for SY-PPV are, on the other hand, not nicely fit by either of the two models, see Fig. 7: the PL declines steeply at relatively large doping densities. The onset of PL quenching at these large doping densities can only be reconciled with the models when exciton diffusion is excluded [see Figs. 7(a) and 7 (b) for $D=0]$. Regarding FRET, a Förster radius $R_{0}$ of maximally $\sim 2.5$ and $\sim 1.5 \mathrm{~nm}$ would then be required for exciton quenching by $\mathrm{p}$ - and n-type doping, respectively. To address the consistency of this hypothesis, $R_{0}$ was calculated from Eq. (2), using the emission spectrum of SY-PPV in Fig. 2. The molar absorption coefficients of the $\mathrm{n}$ - and p-type polarons were determined by either the absorption spectra in Fig. 5 or the absorption spectra measured in reflectance in Fig. S4 in the Supplemental Material [17]. These absorption spectra were taken at known doping densities. The orientation factor $\kappa^{2}$ was again taken to be 0.655 [33] and the refractive index $n$ to be 2 [34]. A fluorescence quantum yield of $17 \%$ was taken from Ref. [26]. The resultant critical radii for $\mathrm{p}$ - and n-type doping were $2.8 \pm 0.6$ and $2.3 \pm 0.4 \mathrm{~nm}$, respectively. This means that the rather large p-type doping density threshold at which PL in SY-PPV is quenched can indeed be explained by both the FRET and the CT modeling in case exciton diffusion is somehow blocked. For n-type doping, an $R_{0}$ value of $2.3 \pm 0.4 \mathrm{~nm}$ would suggest a stronger quenching by FRET than observed in Fig. 7(a), even when diffusion is excluded. However, the sources of possible errors discussed above for MDMO-PPV can also in this case very well lead to this discrepancy.

The sudden drop in PL intensity beyond the onset of quenching may then be related to an enhancement of the exciton diffusion: in case exciton diffusion in SY-PPV rapidly increases around a p-type doping density of $2 \cdot 10^{18} \mathrm{~cm}^{-3}$, a rapid transition between the dashed red line and the solid blue line for, as an example, $R_{0}=2.5 \mathrm{~nm}$ is expected in Fig. 7(a). This would then be in line with the experimentally obtained PL quenching profile for $\mathrm{p}$-type doping in SY-PPV. To substantiate the suggestion of an abrupt increase in exciton diffusion and to provide a possible physical reason, mobility measurements in $\mathrm{n}$ - and p-type doped SY-PPV in an electrochemical transistor structure [see Fig. 8(a)] were performed. The results show a threshold for the doping density dependence of the mobility, as plotted in Fig. 8(b). Interestingly, the thresholds for $\mathrm{n}$ - and p-type doping in Fig. 8(b) coincide quite well with the densities at which the sudden transitions in Fig. 7 occur. Note that the error in experimentally determined doping density is relatively small at these low doping densities. The coincidence of doping density at which PL quenching is initiated and at which the mobility is enhanced suggests a direct connection between charge mobility and trapping and exciton diffusion [36]. Within the lifetime of the exciton, diffusion by polarons is, however, not significant in SY-PPV. From the mobilities in Fig. 8(b) and the Einstein relation, a diffusion constant $D$ between $10^{-10} \mathrm{~cm}^{2} \mathrm{~s}^{-1}$ and $10^{-7} \mathrm{~cm}^{2} \mathrm{~s}^{-1}$ can be estimated. These numbers are small compared to the exciton diffusion constant that is in the range of $10^{-4} \mathrm{~cm}^{2} \mathrm{~s}^{-1}$. Nevertheless, the relatively weak quenching in SY PPV found here (again) confirms the finding that electroluminescence from copolymers is relatively strong compared to their homopolymer counterparts, e.g. MDMO-PPV [37,38].

In principle, the abrupt PL quenching in SY-PPV could also be related to an erroneous determination of the doping 
density. Due to the chemical potential difference at the PEO:LEP interface, an EDL is formed by a small (fraction of the) charging current before actual doping sets in. A difference in chemical potential for the ions in SY-PPV, MDMO-PPV, and P3HT might then result in the different quenching profiles as shown in Fig. 4 . $\mathrm{A} \approx 1$-nm-thick EDL with a potential drop of $1 \mathrm{~V}$ would then correspond to a surface charge density of $3 \cdot 10^{17} \mathrm{~cm}^{-2}$. Such a charge density residing in a $\sim 1 \mathrm{~nm}$ sheet at the interface would correspond to a doping concentration in the entire film $(\sim 100 \mathrm{~nm})$ of a factor $100 / 1=100$ times lower. Since the biases used in the experiment are in the $0-3 \mathrm{~V}$ range, the amount of doping lost to EDL formation of this type is negligible in comparison to the total doping concentration. Hence, the anomalous behavior in SY-PPV compared to MDMO-PPV or P3HT cannot be attributed to a difference in chemical potentials between these materials.

The qualitative behavior of the doping dependence of PL quenching in the experiment and the models (see Fig. 7) confirms on hindsight that the experiment is characterized by homogeneous doping. Successive doping of phase separated regions would lead to a two-step reduction of the PL. In case such an effect is more gradual, then quenching is still expected to be distributed over a larger total injected charge density (horizontal axis of Fig. 7) than in the case of homogeneous doping. The models assume a homogeneous doping density and are able to model the qualitative behavior relatively well (for P3HT and MDMO-PPV). It is in this context important that, for both models, changes in parameter values have little or no effect on the steepness of the slope; hence, an inhomogeneity-induced broadening cannot easily be mapped on (a change in) any of the fitting parameters. A steeper doping density dependence (for SY-PPV) is furthermore not in line with the doping over phase-separated regions as indicated above. Therefore, combined with the discussion in the Experimental Results section, we do see little indication that significant inhomogeneous doping of the conjugated polymer takes place.

The doping densities at which polaron-induced exciton quenching occurs are relevant densities for various types of devices. In efficient polymer LECs, salt densities of $\sim 6 \cdot 10^{19} \mathrm{~cm}^{-3}$ are present in the active layer [39]. In case all salt dissociates into ions, such salt densities lead to highly doped regions that can quench all excitons in case they sit close to the recombination zone. Organic light-emitting diodes operating in the space charge limited regime, on the other hand, generally do not reach the carrier densities found here for exciton quenching. Only when relatively large bias voltages are used to obtain high brightness may the polaron density result in significant exciton quenching [1]. In light-emitting field effect transistors, carrier densities in the range $10^{18}-10^{20} \mathrm{~cm}^{-3}$ pile up near the recombination zone. As a result, exciton-polaron quenching may significantly reduce the efficiency if not prevented by, for example, the introduction of multiple layers to separate carrier transport from carrier recombination [40]. In bulk heterojunction solar cells, polaron densities around $10^{16}$ $\mathrm{cm}^{-3}$ are typically reached under illumination [41]. Therefore, in these cells, polaron-induced exciton quenching is unlikely to lead to a significant reduction in efficiency.

\section{SUMMARY AND CONCLUSION}

The effect of polaron-induced exciton quenching in conjugated polymers has been studied in a solid-state polymer film. By controlled electrochemical doping, the polaron density dependence of the exciton quenching could be determined in several polymers. The density was furthermore confirmed by the occurrence of strong bleaching of the optical absorption. The critical doping density at which exciton quenching occurs ranges between $1 \cdot 10^{18}-2 \cdot 10^{19} \mathrm{~cm}^{-3}$, including the experimental uncertainty, setting an upper limit for the carrier density near and at the recombination zone of efficient emissive devices. The density dependence itself could be modeled by considering exciton diffusion followed by either CT or FRET in a homogeneous density of polarons. Both FRET and CT qualitatively fit the data. Fitting with parameters obtained from literature resulted in reasonable quantitative fits with PL quenching occurring at similar polaron densities. It shows that, for interpreting experimental data related to polaron-induced exciton quenching, (i) exciton diffusion, (ii) CT, and (iii) resonance energy transfer should be considered. Thus, to reduce exciton quenching in devices which convert electrical current in light, the exciton diffusion, the spectral overlap between the emitting exciton and the absorbing polaron, and/or the polaron density need to be reduced.

\section{ACKNOWLEDGMENT}

This work is supported by NanoNextNL, a micro and nanotechnology consortium of the Government of the Netherlands and 130 partners, Project No. NNNL.06D.04.
[1] N. C. Giebink and S. R. Forrest, Phys. Rev. B 77, 235215 (2008).

[2] J. Kalinowski, W. Stampor, J. Szmytkowski, D. Virgili, M. Cocchi, V. Fattori, and C. Sabatini, Phys. Rev. B 74, 085316 (2006).

[3] S. M. King, D. Dai, C. Rothe, and A. P. Monkman, Phys. Rev. B 76, 085204 (2007).

[4] J. M. Hodgkiss, S. Albert-Seifried, A. Rao, A. J. Barker, A. R. Campbell, R. A. Marsh, and R. H. Friend, Adv. Funct. Mater. 22, 1567 (2012).

[5] S. van Reenen, T. Akatsuka, D. Tordera, M. Kemerink, and H. J. Bolink, J. Am. Chem. Soc. 135, 886 (2013).
[6] M. C. Gwinner, D. Kabra, M. Roberts, T. J. K. Brenner, B. H. Wallikewitz, C. R. McNeill, R. H. Friend, and H. Sirringhaus, Adv. Mater. 24, 2728 (2012).

[7] J. Liu, I. Engquist, X. Crispin, and M. Berggren, J. Am. Chem. Soc. 134, 901 (2012).

[8] R. H. Young, C. W. Tang, and A. P. Marchetti, Appl. Phys. Lett. 80, 874 (2002).

[9] H. Z. Lin, S. R. Tabaei, D. Thomsson, O. Mirzov, P. O. Larsson, and I. G. Scheblykin, J. Am. Chem. Soc. 130, 7042 (2008).

[10] G. Li, J. Shinar, and G. E. Jabbour, Phys. Rev. B 71, 235211 (2005).

[11] A. Salleo and R. A. Street, J. Appl. Phys. 94, 471 (2003). 
[12] A. L. Holt, J. M. Leger, and S. A. Carter, J. Chem. Phys. 123, 044704 (2005).

[13] H. Shimotani, G. Diguet, and Y. Iwasa, Appl. Phys. Lett. 86, 022104 (2005).

[14] P. Matyba, K. Maturova, M. Kemerink, N. D. Robinson, and L. Edman, Nat. Mater. 8, 672 (2009).

[15] S. van Reenen, P. Matyba, A. Dzwilewski, R. A. J. Janssen, A. Edman, and M. Kemerink, Adv. Funct. Mater. 21, 1795 (2011).

[16] H. H. P. Gommans, M. Kemerink, G. G. Andersson, and R. M. T. Pijper, Phys. Rev. B 69, 155216 (2004).

[17] See Supplemental Material at http://link.aps.org/supplemental/ 10.1103/PhysRevB.89.205206 for additional experimental and modeling results.

[18] W. C. Germs, J. J. M. van der Holst, S. L. M. van Mensfoort, P. A. Bobbert, and R. Coehoorn, Phys. Rev. B 84, 165210 (2011).

[19] F. Etzold, I. A. Howard, R. Mauer, M. Meister, T. D. Kim, K. S. Lee, N. S. Baek, and F. Laquai, J. Am. Chem. Soc. 133, 9469 (2011).

[20] I. A. Howard, F. Etzold, F. Laquai, and M. Kemerink, Adv. Energy Mater. (2014), doi:10.1002/aenm.201301743.

[21] R. Paulini, K. Muller, and F. Diederich, Angew. Chem., Int. Ed. 44, 1788 (2005).

[22] B. Valeur, Molecular Fluorescence: Principles and Applications (WILEY-VCH, Weinheim, 2001).

[23] U. Gosele, M. Hauser, U. K. A. Klein, and R. Frey, Chem. Phys. Lett. 34, 519 (1975).

[24] A. J. Gesquiere, S. J. Park, and P. F. Barbara, J. Am. Chem. Soc. 127, 9556 (2005).

[25] M. C. Heiber and A. Dhinojwala, J. Phys. Chem. C 117, 21627 (2013).

[26] E. W. Snedden, L. A. Cury, K. N. Bourdakos, and A. P. Monkman, Chem. Phys. Lett. 490, 76 (2010).
[27] D. E. Markov, J. C. Hummelen, P. W. M. Blom, and A. B. Sieval, Phys. Rev. B 72, 045216 (2005).

[28] G. Wilemski and M. Fixman, J. Chem. Phys. 58, 4009 (1973).

[29] P. E. Shaw, A. Ruseckas, and I. D. W. Samuel, Adv. Mater. 20, 3516 (2008).

[30] O. V. Mikhnenko, F. Cordella, A. B. Sieval, J. C. Hummelen, P. W. M. Blom, and M. A. Loi, J. Phys. Chem. B 112, 11601 (2008).

[31] J. Piris, T. E. Dykstra, A. A. Bakulin, P. H. M. van Loosdrecht, W. Knulst, M. T. Trinh, J. M. Schins, and L. D. A. Siebbeles, J. Phys. Chem. C 113, 14500 (2009).

[32] K. M. Gaab and C. J. Bardeen, J. Phys. Chem. A 108, 10801 (2004).

[33] M. A. Stevens, C. Silva, D. M. Russell, and R. H. Friend, Phys. Rev. B 63, 165213 (2001).

[34] J. M. Ziebarth and M. D. McGehee, Appl. Phys. Lett. 83, 5092 (2003).

[35] F. Marchioni, R. Chiechi, S. Patil, F. Wudl, Y. Chen, and J. Shinar, Appl. Phys. Lett. 89, 061101 (2006).

[36] Z. Q. Liang and B. A. Gregg, Adv. Mater. 24, 3258 (2012).

[37] H. Spreitzer, H. Becker, E. Kluge, W. Kreuder, H. Schenk, R. Demandt, and H. Schoo, Adv. Mater. 10, 1340 (1998).

[38] S. Gambino, A. K. Bansal, and I. D. W. Samuel, Org. Electron. 14, 1980 (2013).

[39] S. Tang, J. Pan, H. A. Buchholz, and L. Edman, J. Am. Chem. Soc. 135, 3647 (2013).

[40] R. Capelli, S. Toffanin, G. Generali, H. Usta, A. Facchetti, and M. Muccini, Nat. Mater. 9, 496 (2010).

[41] T. G. J. van der Hofstad, D. Di Nuzzo, S. van Reenen, R. A. J. Janssen, M. Kemerink, and S. C. J. Meskers, J. Phys. Chem. C 117, 3210 (2013). 